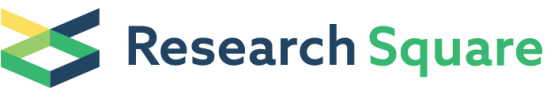

\section{The diagnostic and prognostic value of mature and total adrenomedullin for sepsis: a prospective, observational study}

Tetsu Yonaha ( $\nabla$ tetsu.yonaha@gmail.com )

University of Miyazaki https://orcid.org/0000-0002-4935-2299

\section{Toyoaki Maruta}

University of Miyazaki

Go Otao

University of Miyazaki

Koji Igarashi

TOSOH Corporation

Sayaka Nagata

University of Miyazaki

Takeshi Yano

University of Miyazaki

Masahiko Taniguchi

University of Miyazaki

Kazuo Kitamura

University of Miyazaki

Isao Tsuneyoshi

University of Miyazaki

\section{Research Article}

Keywords: Adrenomedullin, Biomarkers, sepsis, diagnosis, mortality

Posted Date: March 11th, 2021

DOI: https://doi.org/10.21203/rs.2.19873/v2

License: (1) (i) This work is licensed under a Creative Commons Attribution 4.0 International License. Read Full License

Version of Record: A version of this preprint was published at Anaesthesiology Intensive Therapy on January 1st, 2021. See the published version at https://doi.org/10.5114/ait.2021.111345. 


\section{Abstract}

Background: Biologically active adrenomedullin (mature AM) has received considerable attention as a new biomarker of sepsis and septic shock. However, information about this peptide is limited. In this study, we further investigated the value of mature AM for diagnosis and outcome prediction in sepsis.

Methods: This was a prospective, observational, single-centre study. Patients admitted to the intensive care unit (ICU) were retrospectively categorised into non-sepsis or sepsis groups, according to the Sepsis3 definitions. Plasma levels of mature and total (the sum of the levels of intermediate and mature forms) AM were measured, and their usefulness was compared with those of other sepsis biomarkers, such as procalcitonin and presepsin.

Results: Of the 98 patients included in the final analysis, 42 were assigned to the non-sepsis and 56 to the sepsis group. Levels of mature and total AM on admission were significantly higher in patients with sepsis than in those without sepsis. The areas under the receiver operating characteristic curves of mature and total AM for predicting 28-day mortality in patients with sepsis became significant on day 3 after admission. A good correlation between the AM forms was found, indicating that the changes in their plasma levels may directly reflect each other.

Conclusion: Because the levels of mature and total AM increased significantly in patients with sepsis on admission, both forms may be used as reliable and early biomarkers for diagnosing sepsis according to the Sepsis-3 definitions. However, prediction of 28-day mortality in such patients would require several days of ICU stay.

\section{Introduction}

Adrenomedullin (AM) is a potent vasodilator peptide that was first identified in the extracts of human pheochromocytoma [1]. As of date, it is known to be synthesised in several organs and tissues [2]. AM affects the physiological functions of the cardiovascular system, kidneys, and central nervous system. It regulates blood pressure and vascular tone, increases the cardiac output, and promotes diuresis and natriuresis [1-5].

Kitamura et al. [6, 7] were the first to report that two major molecular forms of AM circulate in the human blood, namely the biologically active mature AM (mature AM) form with an amidated C-terminus, and an inactive intermediate AM with a non-amidated C-terminal glycine. Although it is assumed that mature AM might be a key peptide with the AM function, circulating mature AM is quickly degraded and cleared from the circulation and is difficult to detect using a standard immunoassay because of the masking effect of its binding protein (complement factor $\mathrm{H}$ ) [8]. The mid-regional AM (MR-proAM or proAM), which is the inactive fragment of $A M$, is more stable than mature $A M$ and is substituted for speculating the levels of mature AM [9]. In clinical settings, MR-proAM has been identified to be closely associated with the sequential organ failure assessment (SOFA) or acute physiology and chronic health evaluation (APACHE) II scores, and can be useful as a diagnostic and prognostic biomarker for sepsis [9-18]. 
Recently, a double monoclonal sandwich immunoassay was developed that can also measure bioactive mature AM, which is called bio-ADM $[15,19]$. Several investigators have indicated that the levels of mature AM could serve as a useful and objective biomarker to predict severity, organ failure, and mortality in patients with sepsis $[10,20]$. However, further evaluation is required for the clinical use of mature AM as a reliable biomarker of sepsis [8]. Therefore, in this study, we aimed at obtaining additional information about mature AM, including its efficacy and the validation of the optimal cut-off, to help in its development as a marker of sepsis.

\section{Methods}

\section{Study protocol}

This prospective, observational, single-centre study was conducted in accordance with the principles of the Declaration of Helsinki and was approved by the Hospital Ethical Committee for Human Studies (Ref: 0-0317) on December 1, 2014, and retrospectively registered with the Japanese Clinical Trial Registry "UMIN-CTR" (Ref: UMIN000036474). General or written informed consent was obtained from all patients. Strengthening the Reporting of Observational Studies in Epidemiology (STROBE) guidelines were followed in the preparation of this manuscript. For analysis, patients were retrospectively categorized into non-sepsis or sepsis groups, according to the Sepsis-3 definitions [21]. Exclusion criteria were age $<20$ years, pregnancy, trauma, postoperative patients, or lack of informed consent.

\section{Data collection}

Clinical data obtained from electronic medical records included the first diagnosis, chronic comorbidities, laboratory test results, microbiology, and biomarker levels on admission to the intensive care unit (ICU). Blood samples were collected for measuring the levels of AM peptides on ICU admission (day 1) and day 3 post-ICU admission. The blood samples were collected into tubes containing $1.0 \mathrm{mg} \mathrm{mL}^{-1}$ EDTA-2Na and were split into small aliquots to avoid repeated freezing and thawing. The aliquoted samples were frozen as quickly as possible and stored at $-70^{\circ} \mathrm{C}$ until use. AM is derived from an AM precursor via a two-step enzymatic reaction. First, a 185-amino acid AM precursor, known as preproadrenomedullin, is converted to glycine-extended AM, which is the 53-amino acid, inactive, intermediate form of AM (intermediate AM). Subsequently, by enzymatic amidation, intermediate AM is converted to mature AM, a 52-amino acid peptide with an amide group at the $\mathrm{C}$-terminus [6, 7]. Because conventional assays cannot distinguish between intermediate and mature AM forms, AM sometimes indicates total AM, which is the sum of the levels of intermediate and mature forms. In our study, plasma levels of both mature and total AM were measured by a specific fluorescence immunoassay (Tosoh Corporation, Tokyo, Japan) using two independent antibodies-one that binds to the ringed structure was used for the total AM assay and the other that binds to the middle region between the ring and the C-terminal portion of the peptide was used for the mature AM assay, as described previously [22, 23]. Plasma presepsin (PSEP) levels were 
measured with a rapid chemiluminescent enzyme immunoassay (CLEIA) using a PATHFAST immunoanalyzer (Mitsubishi Chemical Medience, Tokyo, Japan). Additionally, we obtained data on procalcitonin (PCT), white blood cells (WBCs), C-reactive protein (CRP), lactate, platelet, and creatinine, which were clinically measured in serum samples at the central laboratory of University of Miyazaki Hospital.

\section{Outcomes}

The primary outcome of the study was the diagnostic performance of biomarkers and SOFA score on ICU admission for sepsis. Secondary outcomes were the time-course of AM levels and the prognostic performance of biomarkers and SOFA score for sepsis.

\section{Statistical analysis}

Statistical analysis was performed using MedCalc 17 (MedCalc Software Bvba, Ostend, Belgium) or JMP 15 (SAS Institute Inc., Cary, NC, USA). Data are expressed as median (IQR: interquartile range) or exact numbers. Categorical data were examined using the chi-square or Fisher's exact test. Continuous variables were compared using the Mann-Whitney U or Kruskal-Wallis test, as appropriate. Receiver operating characteristic (ROC) analysis and comparison of the area under the curve (AUC) were performed to evaluate the accuracy of the biomarkers in diagnosing and predicting sepsis. A value of $\mathrm{P}<$ 0.01 was considered statistically significant.

\section{Results}

\section{Diagnosis of sepsis on ICU admission}

Ninety-eight patients were enrolled in this study between January 2015 and November 2018 (Table 1). A total of 56 patients were diagnosed with sepsis according to the Sepsis-3 definitions, and 42 were not diagnosed with sepsis. The abdomen and respiratory tract were the most common sites of infection, whereas heart failure was the most common reason for ICU admission in patients with no sepsis. We could not track the outcome for one patient with sepsis who was transferred to another hospital. The levels of AM peptides, values of clinical parameters including laboratory results, and SOFA score on ICU admission are presented in Table 2. The levels of mature AM, total AM, PCT, and PSEP were substantially higher in patients with sepsis than in those who did not have sepsis on day 1 (Figure 1 and Table 2). However, these significant differences in the levels of both the AM peptides disappeared on day 3 (Figure 1).

To compare the diagnostic accuracy of sepsis using each biomarker, we performed an ROC analysis and determined the AUCs using the values obtained for the 98 patients on ICU admission (Table 3). The AUCs 
of mature and total AM were 0.848 and 0.879 , respectively, indicating that both the AM peptides exhibit significant predictive values for the diagnosis of sepsis, in comparison with other biomarkers, such as PCT, PSEP, SOFA score, and lactate levels $(0.828,0.682,0.772$, and 0.660 , respectively). The cut-off levels of mature and total AM for the diagnosis of sepsis were $5.2 \mathrm{pmol} \mathrm{L}^{-1}\left(\approx 31.2 \mathrm{pg} \mathrm{mL}^{-1}\right)$ and $48.3 \mathrm{pmol} \mathrm{L}^{-1}$ $\left(\approx 289.8 \mathrm{pg} \mathrm{mL}^{-1}\right)$, respectively (Table 3 ). Significant correlations between the levels of mature and total AM were found in blood samples, obtained from the same patients on days 1 and 3 (Figure 2).

The 28-day mortality rates in patients without or with sepsis were $14.3 \%$ and $30.9 \%$, respectively (Table 1). In patients with sepsis, the levels of mature and total AM were not statistically significant on day 1 ; however, they were significantly increased in non-survivors on day 3 compared with the levels in survivors (Figure 3). Likewise, the AUCs of these AM peptides for predicting the 28-day mortality rates in patients with sepsis became significant on day 3 but not on day 1 (Table 4). The cut-off levels of mature and total AM for the 28-day mortality on day 3 were $5.4 \mathrm{pmol} \mathrm{L}^{-1}\left(\approx 32.4 \mathrm{pg} \mathrm{mL}^{-1}\right)$ and $72.5 \mathrm{pmol} \mathrm{L}^{-1}(\approx 435.0 \mathrm{pg}$ $\mathrm{mL}^{-1}$ ), respectively (Table 4).

\section{Discussion}

In this study, we found that the plasma levels of mature and total AM on day 1 of ICU admission in patients with sepsis were significantly higher than in those without sepsis. The good correlation between the plasma levels of both the peptides recognized in the present study demonstrates that both total and mature AM are equally useful in diagnosing sepsis based on the latest Sepsis-3 definitions. On the contrary, with regard to the clinical outcome in patients with sepsis, a significant difference in the level of each AM was recognized between survivors and non-survivors 3 days after the admission. In addition, the levels of both the AM forms could predict the 28-day mortality in patients with sepsis on day 3 , but not on day 1 . Therefore, unlike in previous studies, we found that it may take several days for these AM peptides to prognosticate the 28-day mortality in patients with sepsis, because AM levels increased even in patients with a good prognosis on day 1 .

In some previous studies, the levels of total AM were assessed and their utility in diagnosing sepsis was compared with those of other chemical biomarkers, such as CRP, PCT, lactate, and PSEP [8, 9, 18, 20, 2426]. We found that among all the clinical parameters examined, the diagnostic accuracy for sepsis on the first day of ICU admission was the highest for total AM, and that of mature AM was the second highest. It is assumed that mature AM is biologically active, and would thus be a more informative biomarker than total AM in patients with sepsis. In our study, the cut-off level of mature AM for the diagnosis of sepsis was approximately $31.2 \mathrm{pg} \mathrm{mL}^{-1}$, and its AUC was 0.848 , with a sensitivity of $88.1 \%$ and a specificity of $67.9 \%$. Other investigators have also reported that the ability of mature AM to identify patients with sepsis was modest because an AUC of 0.76 and a Youden's index derived cut-off of $37 \mathrm{pg} \mathrm{mL}^{-1}$ generated a sensitivity of $61 \%$ and a specificity of $80 \%$ [20]. These cut-off values of mature AM were close, indicating that the values of about $30 \mathrm{pg} \mathrm{mL}^{-1}$ could be applicable to diagnose sepsis, which is a novel finding for mature AM. 
The half-life of mature AM in the plasma is approximately $20 \mathrm{~min}$. As a biomarker of acute cardiovascular failure, the levels of mature AM can be significantly changed during the first $24 \mathrm{~h}$ after diagnosis [10]. Indeed, in our study, the levels of mature AM in patients with sepsis significantly increased on day 1 but reverted to the same levels as in patients without sepsis, 3 days after admission, indicating that a time frame in which sepsis can be identified by this peptide may be confined to an early stage after the onset.

Accumulating evidence indicates that the clinical outcome in patients with sepsis can be diagnosed based on AM levels [10-12, 14-17]. However, investigators have pointed to the need for more data to evaluate the mortality rate in patients with sepsis prognosticated by the levels of mature AM [8]. Marino et al. [15] demonstrated that the levels of mature AM at ICU admission strongly correlated with the 28-day mortality rate in patients with sepsis. Patients with AM levels $>70 \mathrm{ng} \mathrm{L}^{-1}$ had a 28 -day survival rate of $55 \%$. Recent evidence supports this result. Kim et al. [10] reported that the cut-off value of mature AM levels for 28-day mortality was $97.12 \mathrm{pg} \mathrm{mL}^{-1}$. However, in the present study, the levels of total and mature AM on day 1 could not be used to predict the 28-day sepsis-related mortality. It is well known that AM levels increase in patients with heart failure, myocardial infarction, pulmonary hypertension, SIRS, inflammatory bowel diseases, and renal failure, resulting in increased AM levels even in patients with sepsis having a good prognosis [2, 27-30]. This notion can explain our results presented in Figure 3 , which indicates that the levels of mature and total AM were not significantly different between nonsurvivors and survivors on day 1 .

We found that the levels of mature and total AM on day 3 in patients with sepsis had a high predictive value for sepsis-related mortality as determined by ROC analysis. Interestingly, Mebazaa et al. [11] reported that in patients with the levels of mature $A M>70 \mathrm{pg} \mathrm{mL}^{-1}$ on admission, a decrease in its levels below $70 \mathrm{pg} \mathrm{mL}^{-1}$ on day 2 was associated with the recovery of organ function on day 7 and a better 28day outcome ( $9.5 \%$ mortality), whereas persistently elevated levels of mature AM on day 2 were associated with prolonged organ dysfunction and high 28-day mortality (38.1\% mortality). These results suggest that the levels of mature AM may remain high if the treatment is unsuccessful, and organ damage continues in sepsis. Indeed, as demonstrated in Figure 3, the sustained increase in the levels of mature AM in patients with sepsis correlated with worsening prognoses.

In the present study, the cut-off levels of mature and total AM on day 3 for the diagnosis of the 28-day sepsis-related mortality were approximately 32.4 and $435.0 \mathrm{pg} \mathrm{mL}^{-1}$, respectively. The cut-off level of mature AM was lower than that in previous studies $\left(30 \mathrm{pg} \mathrm{mL}^{-1}\right.$ versus $\left.70 \mathrm{pg} \mathrm{mL}^{-1}\right)$. This could be explained by the sensitivity of the assay used in each study, indicating that the cut-off values obtained by different assay methods may be different. Recently, Lundberg et al. [20] reported a Yuden's index cut-off of $45 \mathrm{pg} \mathrm{mL}^{-1}$ for predicting the 28-day sepsis-related mortality in their sepsis cohort, which was as low as in the present study.

It has been presumed that total AM represents the sum of synthesized intermediate AM and mature AM. However, little is known about the ratio of each peptide contained in total AM. We suspected that if the 
composition ratio would vary for each patient with sepsis, the levels of total AM could not directly reflect the functional status of mature AM [31]. However, as discussed previously, we found a definite correlation between the levels of mature and total AM, both of which were measured from an identical blood sample, with a resultant increase in a similar fashion. Based on our results, it may be sufficient to measure either mature or total AM for the diagnosis of sepsis.

This study has some limitations. First, this study was performed as a small, single-centre study focusing on the diagnostic performance of AM for sepsis and septic shock based on the Sepsis-3 definitions. Given the similarities in responses and outcomes, such a study will likely require a much larger patient sample size and would potentially be best accomplished with a multi-centre design. Second, as discussed previously, the AM-binding protein, complement factor $\mathrm{H}$, hinders the reliable measurement of mature AM $[9,18,32]$; this technical difficulty in the assay precludes its practical use. Recently, Weber et al. [19] reported a new assay to reliably measure the bio-active AM using a double monoclonal sandwich immunoassay that can measure C-terminally amidated biologically active AM. We used our originally developed assay for measuring mature AM and found that the limits of detection and quantitation were 0.133 and $0.085 \mathrm{pmol} \mathrm{L}^{-1}$, respectively, according to the Clinical and Laboratory Standards Institute (CLSI) protocols. The intra- and inter-assay coefficients of variation were $1.8 \%$ and $5.1 \%$, respectively [23]. Thus, we believe our assay is as reliable as the one reported by Weber et al. Third, we do not provide any evidence of whether AM might resolve or worsen the septic state itself. Although the effects of AM or AM antibody therapy for sepsis have been evaluated in some preclinical animal studies [33], it is still unclear whether AM will have beneficial therapeutic effects in patients with sepsis. There is an ongoing, multicentre study evaluating the AM-binding antibody therapy for sepsis (AdrenOSS-2) [34]. The results of this study might eventually be useful in characterizing the efficacy of AM therapy in patients with sepsis.

\section{Conclusions}

We conclude that (1) at the early onset of sepsis, both mature and total AM are excellent biomarkers with the highest diagnostic accuracy for sepsis among the biomarkers that we evaluated; (2) a good correlation exists between each AM, indicating that the changes in the plasma levels may directly reflect one another; and (3) both AM peptides have the potential to predict the 28-day mortality in patients with sepsis several days after the onset. Further studies are required to clarify the relationship between mature $\mathrm{AM}$ and its clinical significance in comparison with total AM.

\section{Acknowledgements}

1. Financial support and sponsorship: This study was supported by a grant for clinical research from the University of Miyazaki Hospital.

2. Conflicts of interest: $\mathrm{KI}$ is an employee of Tosoh Corporation. The other authors declare no competing interests. 
3. The authors would like to thank Mariko Tokashiki, Noriko Hidaka, Mio Kurogi, and Toshiko Watanabe for their technical advice and assistance in data collection. We also thank Editage (www.editage.com) for English language editing.

\section{References}

1. Kitamura K, Kangawa K, Kawamoto $\mathrm{M}$, et al. Adrenomedullin: a novel hypotensive peptide isolated from human pheochromocytoma. Biochem Biophys Res Commun 1993; 192: 553-5 doi: 10.1006/bbrc.1993.1451.

2. Kato J, Kitamura K. Bench-to-bedside pharmacology of adrenomedullin. Eur J Pharmacol 2015; 764: 140-14 doi: 10.1016/j.ejphar.2015.06.061.

3. Ebara T, Miura K, Okumura $\mathrm{M}$, et al. Effect of adrenomedullin on renal hemodynamics and functions in dogs. Eur J Pharmacol 1994; 263: 69-73. doi: 10.1016/0014-2999(94)90524-x.

4. Yamaguchi T, Baba K, Doi Y, Yano K. Effect of adrenomedullin on aldosterone secretion by dispersed rat adrenal zona glomerulosa cells. Life Sci 1995; 56: 379-3 doi: 10.1016/0024-3205(94)00903-1.

5. Fukuhara M, Tsuchihashi T, Abe I, Fujishima M. Cardiovascular and neurohormonal effects of intravenous adrenomedullin in conscious rabbits. Am J Physiol 1995; 269: R1289-1293. doi: 10.1152/ajpregu.1995.269.5.R1289.

6. Kitamura K, Sakata J, Kangawa K, Kojima M, Matsuo H, Eto T. Cloning and characterization of cDNA encoding a precursor for human adrenomedullin. Biochem Biophys Res Commun 1993; 194: 720-72 doi: 10.1006/bbrc.1993.1881.

7. Kitamura K, Kato J, Kawamoto M, et al. The intermediate form of glycine-extended adrenomedullin is the major circulating molecular form in human plasma. Biochem Biophys Res Commun 1998; 244: 551-555. doi: 10.1006/bbrc.1998.8310.

8. Kim MH, Choi JH. An update on sepsis biomarkers. Infect Chemother 2020; 52: 1-18. doi: 10.3947/ic.2020.52.1.1.

9. Önal U, Valenzuela-Sánchez F, Vandana KE, Rello J. Mid-regional pro-adrenomedullin (MR-proADM) as a biomarker for sepsis and septic shock: narrative review. Healthcare (Basel) 2018; 6: 110. doi: 10.3390/healthcare6030110.

10. Kim H, Hur M, Struck J, Bergmann A, Di Somma S. Circulating biologically active adrenomedullin predicts organ failure and mortality in sepsis. Ann Lab Med 2019; 39: 454-4 doi: 10.3343/alm.2019.39.5.454.

11. Mebazaa A, Geven C, Hollinger A, et al. Circulating adrenomedullin estimates survival and reversibility of organ failure in sepsis: the prospective observational multinational Adrenomedullin and Outcome in Sepsis and Septic Shock-1 (AdrenOSS-1) study. Crit Care 2018; 22: 354. doi: 10.1186/s13054-018-2243-2.

12. Caironi P, Latini R, Struck J, et al. Circulating biologically active adrenomedullin (bio-ADM) predicts hemodynamic support requirement and mortality during sepsis. Chest 2017; 152: 312-3 doi: 
10.1016/j.chest.2017.03.035.

13. Gonzalez Del Castillo J, Wilson DC, Clemente-Callejo C, et al. Biomarkers and clinical scores to identify patient populations at risk of delayed antibiotic administration or intensive care admission. Crit Care 2019; 23: 335. doi: 10.1186/s13054-019-2613-4.

14. Saeed K, Wilson DC, Bloos F, et al. The early identification of disease progression in patients with suspected infection presenting to the emergency department: a multi-centre derivation and validation study. Crit Care 2019; 23: 40. doi: 10.1186/s13054-019-2329-5.

15. Marino R, Struck J, Maisel AS, Magrini L, Bergmann A, Di Somma S. Plasma adrenomedullin is associated with short-term mortality and vasopressor requirement in patients admitted with sepsis. Crit Care 2014; 18: R34. doi: 10.1186/cc13731.

16. Chen YX, Li CS. The predictive value of adrenomedullin for development of severe sepsis and septic shock in emergency department. Biomed Res Int 2013; 2013: 960101. doi: 10.1155/2013/960101.

17. Chen YX, Li CS. Prognostic value of adrenomedullin in septic patients in the ED. Am J Emerg Med 2013; 31: 1017- doi: 10.1016/j.ajem.2013.03.017.

18. Bernal-Morell E, García-Villalba E, Vera MDC, et al. Usefulness of midregional pro-adrenomedullin as a marker of organ damage and predictor of mortality in patients with sepsis. J Infect 2018; 76: 2492 doi: 10.1016/j.jinf.2017.12.003.

19. Weber J, Sachse J, Bergmann S, Sparwaßer A, Struck J, Bergmann A. Sandwich immunoassay for bioactive plasma adrenomedullin. J Appl Lab Med 2017; 2: 222-2 doi: 10.1373/jalm.2017.023655.

20. Lundberg $\mathrm{OHM}$, Lengquist $M$, Spångfors $M$, et al. Circulating bioactive adrenomedullin as a marker of sepsis, septic shock and critical illness. Crit Care 2020; 24: 636. doi: 10.1186/s13054-020-03351-1.

21. Singer M, Deutschman CS, Seymour CW, et al. The Third International Consensus Definitions for Sepsis and Septic Shock (Sepsis-3). JAMA 2016; 315: 801-810. 10.1001/jama.2016.0287.

22. Nomura I, Kato J, Tokashiki M, Kitamura K. Increased plasma levels of the mature and intermediate forms of adrenomedullin in obesity. Regul Pept 2009; 158: 127-1 doi:

10.1016/j.regpep.2009.08.003.

23. Kawano S, Kawagoe Y, Kuwasako K, et al. Gender-related alterations in plasma adrenomedullin level and its correlation with body weight gain. Endocr Connect 2015; 4: 43-4 doi: 10.1530/EC-14-0131.

24. Bernal-Morell E, García-Villalba E, Vera MDC, et al. Usefulness of midregional pro-adrenomedullin as a marker of organ damage and predictor of mortality in patients with sepsis. J Infect 2018; 76: 2492 doi: 10.1016/j.jinf.2017.12.003.

25. Wacker C, Prkno A, Brunkhorst FM, Schlattmann P. Procalcitonin as a diagnostic marker for sepsis: a systematic review and meta-analysis. Lancet Infect Dis 2013; 13: 426-4 doi: 10.1016/S14733099(12)70323-7.

26. Kondo Y, Umemura Y, Hayashida K, Hara Y, Aihara M, Yamakawa K. Diagnostic value of procalcitonin and presepsin for sepsis in critically ill adult patients: a systematic review and meta-analysis. $\mathrm{J}$ Intensive Care 2019; 7: 22. doi: 10.1186/s40560-019-0374-4. 
27. Bunton DC, Petrie MC, Hillier C, Johnston F, McMurray JJV. The clinical relevance of adrenomedullin: a promising profile? Pharmacol Ther 2004; 103: 179- doi: 10.1016/j.pharmthera.2004.07.002.

28. Nishikimi T. Adrenomedullin in the kidney-renal physiological and pathophysiological roles. Curr Med Chem 2007; 14: 1689-16 doi: 10.2174/092986707780830943.

29. Yuyun MF, Narayan HK, Ng LL. Prognostic significance of adrenomedullin in patients with heart failure and with myocardial infarction. Am J Cardiol 2015; 115: 986-9 doi:

10.1016/j.amjcard.2015.01.027.

30. Self WH, Storrow $A B$, Hartmann 0 , et al. Plasma bioactive adrenomedullin as a prognostic biomarker in acute heart failure. Am J Emerg Med 2016; 34: 257-2 doi: 10.1016/j.ajem.2015.10.033.

31. Cao, YN, Kitamura K, Ito K, et al. Glycine-extended adrenomedullin exerts vasodilator effect through amidation in the rat aorta. Regul Pept 2003; 113: 109-114. doi: 10.1016/s0167-0115(03)00002-8.

32. Di Somma S, Magrini L, Travaglino F, et al. Opinion paper on innovative approach of biomarkers for infectious diseases and sepsis management in the emergency department. Clin Chem Lab Med 2013; 51: 1167-11 doi: 10.1515/cclm-2012-0795.

33. Geven C, Kox M, Pickkers P. Adrenomedullin and adrenomedullin-targeted therapy as treatment strategies relevant for sepsis. Front Immunol 2018; 9: 292. doi: 10.3389/fimmu.2018.00292.

34. Geven C, Blet A, Kox M, et al. A double-blind, placebo-controlled, randomised, multicentre, proof-ofconcept and dose-finding phase II clinical trial to investigate the safety, tolerability and efficacy of adrecizumab in patients with septic shock and elevated adrenomedullin concentration (AdrenOSS-2). BMJ Open 2019; 9: e024475. doi: 10.1136/bmjopen-2018-024475.

\section{Abbreviations}

AM: Adrenomedullin; ICU: Intensive care unit; ROC: Receiver operating characteristic; AUC: Area under the curve; PCT: Procalcitonin; PSEP: Presepsin; SOFA: Sequential organ failure assessment; CRP: $C$ reactive protein; MAP: Mean atrial pressure; SIRS: Systemic inflammatory response syndrome; MR-proADM: Midregion of adrenomedullin; APACHE II: Acute physiology and chronic health evaluation II; WBC: White blood cell; PT-INR: Prothrombin time-international normalized ratio; TREM-1: Triggering receptor expressed on myeloid cells 1; HMGB1: High mobility group box 1.

\section{Tables}

Table 1. Clinical characteristics of patients admitted to ICU by Sepsis-3 classification 


\begin{tabular}{|l|c|c|c|}
\hline & Non-sepsis (n=42) & Sepsis (n=56) & $P$-value \\
\hline Age (y), median (IQR) & $66.5(56.0-76.0)$ & $69.0(58.5-78.0)$ & 0.48 \\
Male, n (\%) & $32(76)$ & $37(66)$ & 0.27 \\
Diagnosis, n (\%) & & & $<0.01$ \\
Abdominal disorder & $8(19)$ & $16(29)$ & \\
Abscess & $1(2)$ & $2(4)$ & \\
Aortic disorder & $3(7)$ & $0(0)$ & \\
Blood stream infection & $0(0)$ & $8(14)$ & \\
Heart failure & $10(24)$ & $0(0)$ & \\
Neurological disorder & $1(2)$ & $2(4)$ & \\
Respiratory disorder & $8(19)$ & $15(27)$ & \\
Skin/Soft tissue disorder & $3(7)$ & $4(7)$ & \\
Urinary disorder & $3(7)$ & $5(9)$ & \\
Others & $5(12)$ & $4(7)$ & \multirow{2}{*}{0.05} \\
28-day mortality, n (\%) & $6(14)$ & $17\left(31^{*}\right)$ & \\
\hline
\end{tabular}

* One patient could not be tracked for the outcome.

Table 2. Clinical parameters of patients on ICU admission (day 1)

\begin{tabular}{|c|c|c|c|}
\hline Clinical parameters & Non-sepsis $(n=42)$ & \begin{tabular}{l|l}
$42)$ & Sepsis $(\mathrm{n}=56)$
\end{tabular} & $P$-value \\
\hline Mature AM (pmol L-1) & $2.2(1.2-3.7)$ & $7.8(3.7-18.1)$ & $<0.001$ \\
\hline Total AM (pmol L-1) & $19.0(10.9-35.8)$ & $1 \phi 4(43.3-267)$ & $<0.001$ \\
\hline Procalcitonin $\left(\mathrm{mg} \mathrm{L}^{-1}\right)$ & $0.3(0.1-1.6)$ & 12.6( & $<0.001$ \\
\hline Prese & 563 (298.5-1231) & $1300(492-2483)$ & 0.004 \\
\hline SOFA score & $6(3-8)$ & $10(7-12)$ & $<0.001$ \\
\hline Lactate $\left(\mathrm{mmol} \mathrm{L}^{-1}\right)$ & $1.3(1.0-2.2)$ & 2.3 & 0.007 \\
\hline WBC $\left(\mathrm{mL}^{-1}\right)$ & $0 \not 500$ (8125-1452! & 9900 (3650-15950) & 0.376 \\
\hline $\mathrm{CRP}\left(\mathrm{mg} \mathrm{L}^{-1}\right)$ & $0.39(0.08-1.08)$ & $1 . \$ 3(0.89-2.66)$ & $<0.001$ \\
\hline Platelet $\left(\times 10^{9} \mathrm{~L}^{-1}\right)$ & $178(111-203)$ & $109(34-165)$ & 0.001 \\
\hline Creatinine $\left(\mathrm{mg} \mathrm{dL}^{-1}\right)$ & $0.9(0.7-2.1)$ & $1.6(1.0-2.8)$ & 0.021 \\
\hline
\end{tabular}

Table 3. Diagnostic performance of different clinical parameters on ICU admission (day 1) for sepsis 


\begin{tabular}{|c|c|c|c|c|c|}
\hline $\begin{array}{c}\text { Clinical } \\
\text { parameters }\end{array}$ & Cut-off & AUC (95\% CI) & $\begin{array}{c}\text { Sensitivity } \\
(\%)\end{array}$ & $\begin{array}{c}\text { Specificity } \\
(\%)\end{array}$ & $P$-value \\
\hline Mature AM & $5.2 \mathrm{pmol} \mathrm{Q}=84$ & 8 (0.773- & 88.1 & 67.9 & $\stackrel{<}{001}$ \\
\hline Total AM & $48.3 \underset{1}{\mathrm{pmo}} 0_{\mathrm{L}} 8 \mathrm{~T}$ & $\begin{array}{c}9.9214) \\
0.945)\end{array}$ & 85.7 & 73.2 & $\begin{array}{l}0.001 \\
< \\
0.001\end{array}$ \\
\hline Procalcitonin & $5.21 \mathrm{mg} \mathbb{1}^{-0.82}$ & $8(0.746-$ & 94.6 & 58.9 & $\stackrel{<}{0.001}$ \\
\hline Presepsin & 1315 ng $0-68$ & $2(0.570-$ & 88.9 & 49.1 & 0.002 \\
\hline SOFA score & $8 \quad 0.77$ & $2(0.679-$ & 83.3 & 60.0 & $<\stackrel{<}{0}$ \\
\hline Lactate & $1.8 \mathrm{mmol}_{1} \mathrm{Q} .66$ & $\begin{array}{l}0(0.549- \\
0.771)\end{array}$ & 69.1 & 66.1 & 0.005 \\
\hline WBC & $4500 / \mathrm{mL} 0^{0.155}$ & $3(0.437-$ & 97.6 & 30.9 & 0.037 \\
\hline CRP & $0.534 \mathrm{mg} 9.76$ & $4 \begin{array}{l}(0.663- \\
0.865)\end{array}$ & 66.7 & 83.6 & 0.001 \\
\hline Platelet & $134 \times 10_{1}^{90_{\mathrm{E}} \mathrm{G}}$ & $\begin{array}{l}3(0.586- \\
0.800)\end{array}$ & 70.7 & 67.3 & 0.004 \\
\hline Creatinine & $0.92 \underset{1}{\mathrm{mg}} \mathrm{d} \mathrm{P}^{6} 63$ & $\begin{array}{ll}8 & (0.522- \\
0.755)\end{array}$ & 51.2 & 78.2 & 0.020 \\
\hline
\end{tabular}

Table 4. Prognostic performance (predicting 28-day mortality) of adrenomedullin (AM) in septic patients

\begin{tabular}{|c|c|c|c|c|c|c|}
\hline Biomarkers & Day & $\begin{array}{c}\text { Cut-off (pmol L- } \\
{ }_{1} \text { ) }\end{array}$ & AUC (95\% CI) & $\begin{array}{c}\text { Sensitivity } \\
(\%)\end{array}$ & $\begin{array}{c}\text { Specificity } \\
(\%)\end{array}$ & $\begin{array}{c}P- \\
\text { value }\end{array}$ \\
\hline \multirow[t]{2}{*}{ Mature AM } & 1 & 4.7 & $0.551(0.411-0.685)$ & 88.2 & 39.5 & 0.538 \\
\hline & 3 & 5.4 & $0.892(0.714-1.000)$ & 83.3 & 88.2 & $\stackrel{<}{0.001}$ \\
\hline \multirow[t]{2}{*}{ Total AM } & 1 & 52.5 & $0.644(0.503-0.768)$ & 88.2 & 39.5 & 0.067 \\
\hline & 3 & 72.5 & $0.931(0.791-1.000)$ & 83.3 & 100 & $\begin{array}{l}< \\
0.001\end{array}$ \\
\hline
\end{tabular}

\section{Figures}


Figure 1
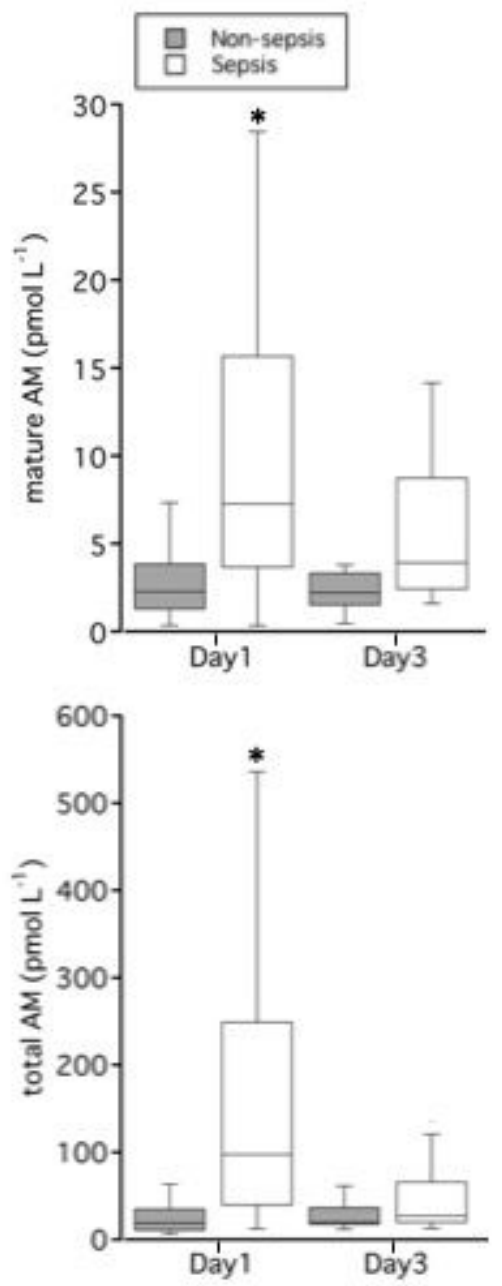

\section{Figure 1}

Comparative plasma levels of mature and total adrenomedullin (AM) between patients with and without sepsis. 
Figure 2
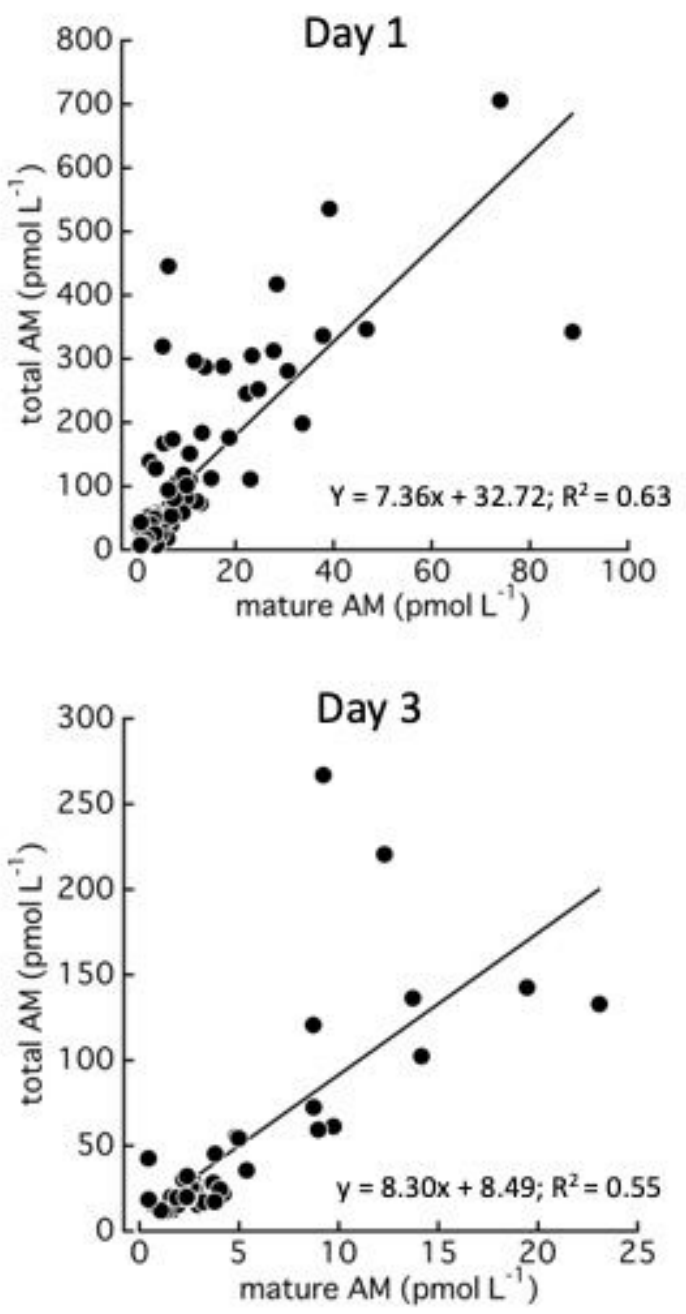

Figure 2

The relationships between mature and total adrenomedullin (AM) on admission to the intensive care unit (day 1 ) and on day 3. 
Figure 3
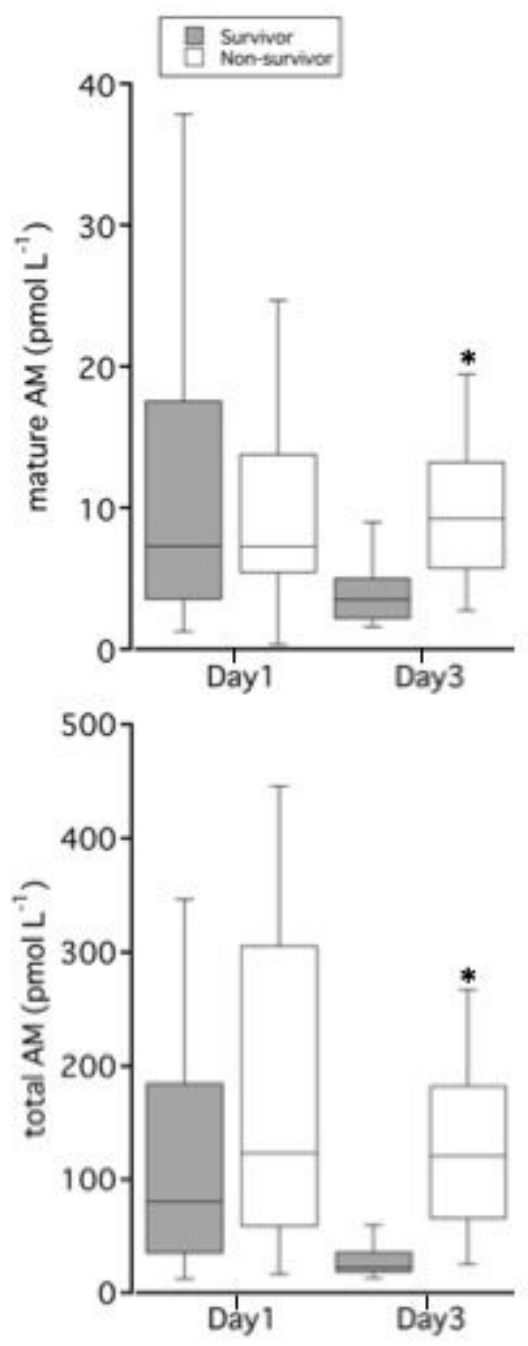

Figure 3

Comparative plasma levels of mature and total adrenomedullin (AM) between survivor and non-survivor patients with sepsis. 